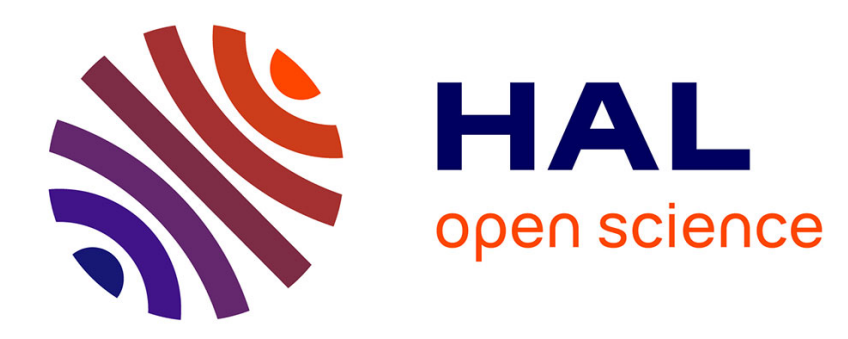

\title{
L'approche par les capacités comme registre des restructurations: un nouveau regard sur l'entreprise et le contrat de travail?
}

Claude Didry

\section{To cite this version:}

Claude Didry. L'approche par les capacités comme registre des restructurations: un nouveau regard sur l'entreprise et le contrat de travail ?. 2012. halshs-00688733

\section{HAL Id: halshs-00688733 \\ https://shs.hal.science/halshs-00688733}

Preprint submitted on 18 Apr 2012

HAL is a multi-disciplinary open access archive for the deposit and dissemination of scientific research documents, whether they are published or not. The documents may come from teaching and research institutions in France or abroad, or from public or private research centers.
L'archive ouverte pluridisciplinaire HAL, est destinée au dépôt et à la diffusion de documents scientifiques de niveau recherche, publiés ou non, émanant des établissements d'enseignement et de recherche français ou étrangers, des laboratoires publics ou privés. 


\title{
L'approche par les capacités comme registre des restructurations : un
} nouveau regard sur l'entreprise et le contrat de travail ?

\author{
Claude Didry \\ IDHE
}

\begin{abstract}
Résumé : Dans la perspective de Sen, l' «approche par les capacités» désigne un principe de gouvernement démocratique fondant les choix publics sur l'évaluation des capacités individuelles, en identifiant des urgences sociales telles que le chômage, à partir des atteintes aux capacités qui en résultent. Il s'agit, dans ce texte, de voir en quoi une telle « approche par les capacités » se retrouve dans les pratiques des représentants des salariés au cours des situations de restructuration. L'évolution de ces pratiques fait en effet apparaître un intérêt nouveau pour les choix des salariés, à travers la signature d' «accords de méthode » ou de GPEC qui intègrent une ouverture sur les projets individuels et sur le devenir de l'entreprise. Elle suggère l'émergence d'une «approche par les capacités » conduisant à envisager l'entreprise à partir de l'apport en capacités que dessine le contrat de travail. Ainsi, l' «approche par les capacités » désigne un registre d'action collective qui, au-delà d'un registre de la critique focalisé sur la défense de l'emploi, tente de lier choix individuels et choix collectifs en esquissant ainsi une «capacité politique » des salariés à travers leur engagement dans la définition de ce que recoupe, à leurs yeux, l'entreprise.
\end{abstract}

En montrant l'étendue de la souffrance liée au chômage qui, au-delà de la perte de revenu compensée parfois par une indemnisation, affecte tous les aspects de la vie du chômeur (sa santé, sa vie familiale, ses motivations professionnelles,sa vie civique), Sen (1997, p. 175-176) suggère que le travail est décisif pour la «capacité » d'un individu comme « liberté réelle que l'on a de choisir entre différents modes de vie ${ }^{1}$ » (Sen 2010, p. 279). Mais il suppose un régime de liberté, l'existence d'individus en bonne santé et suffisamment formés, pour que le travail soit le résultat d'un choix et non, comme dans les différentes formes de travail contraint évoquées par Sen (esclavage aux Etats-Unis, affectation administrative dans les ex-pays communistes), une source d'oppression s'ajoutant aux sujétions traditionnelles des individus ${ }^{2}$. Il en résulte que, dans le travail, les individus engagent leur «capacité », constitutive de ce que Marx définit comme leur «force de travail », c'est-à-dire «le résumé de toutes les capacités physiques et intellectuelles qui existent dans la corporéité, la personnalité vivante d'un être humain, et qu'il met en mouvement chaque fois qu'il produit des valeurs d'usage quelconques. » (Marx 1993, p. 188).

${ }^{1}$. Dans les ouvrages de Sen, la notion de capacité correspond de manière générale à la liberté de choisir sa vie, mais au sens de la préservation, pour un individu, de la liberté de choix dans sa vie, de continuer à vivre comme il choisit de le faire et non comme un choix solennel qui enfermerait le reste de la vie dans un projet irrévocable.

${ }^{2}$. Dont l'exemple, récurrent dans les œuvres de Sen, est l'assignation des femmes aux tâches familiales dans une société patriarcale. 
Compte tenu de cette dimension fondamentale du travail pour la capacité des individus, 1' " approche par les capacités » est, pour Amartya Sen (1997, 2000 et 2003b) la base d'une critique radicale des politiques économiques en Europe, dans un contexte de chômage durablement élevé. En effet, l'approche par les capacités montre de manière immédiate dans le cas du chômage, l'importance qu'il y a à se concentrer « sur la vie humaine et pas seulement sur des «objets de confort» comme les revenus ou les produits de base souvent érigés en critères principaux du succès humain, notamment dans l'analyse économique »(Sen 2010, p. 286). Elle a conduit Sen à mettre en cause ce qu'il nomme 1' « extrémisme budgétaire » qui, en Europe, érige l'équilibre des comptes publics en priorité, au détriment d'une urgence sociale telle que le chômage (Sen 2003b). Dans le même temps, elle a été la base d'une critique de stratégies syndicales focalisées sur la défense des salariés les plus stables en suggérant, sans abandonner la défense des droits acquis, une ouverture sur le devenir des chômeurs (Sen 2000).

Cette critique des politiques macro-économiques et des stratégies syndicales a trouvé un écho dans l'accent mis, au niveau européen, sur la recherche d'une «flexicurité » ou dans l'orientation de syndicats français vers la « sécurisation des parcours professionnels » (CFDT) ou la «sécurité sociale professionnelle » (CGT). Il s'agit, dès lors, au-delà d'une défense de l'emploi par des politiques économiques ou des stratégies syndicales, d'envisager simultanément le devenir de ces derniers. Mais dans un contexte de crise s'accompagnant d'une multiplication des restructurations, une telle orientation visant à faire face en priorité à la montée du chômage ne risque-t-elle pas d'occulter l'importance des choix opérés par les entreprises dans la situation de l'emploi ?

Comme dans le cas du chômage et de l'emploi au niveau de la collectivité nationale, les restructurations font apparaître la dimension collective des choix dans l'entreprise à partir d'un ensemble de procédures d'information et de consultation des représentants des salariés par les directions. En France, elles conduisent fréquemment à des débats et des conflits sociaux dans lesquels la mobilisation du droit permet aux salariés et à leurs représentants d'exprimer leur opposition à des projets de suppression d'emplois et, ainsi, d'arriver à ébranler une gouvernance par «des indicateurs clés en main», tels que, en premier lieu, la rentabilité de l'entreprise (Didry 1998). Les procédures de licenciement collectif constituent ainsi la base d'une «action collective organisée » (Mathieu 2012) donnant lieu, dans un premier temps, à l'expression d'un «registre critique» tant dans les échanges des représentants du personnel au sein des institutions représentatives du personnel et devant la justice, que dans les différentes formes de mobilisation collective des salariés. Mais, depuis 
une décennie, la construction d'une « cause de l'emploi » sur la base d'une critique des choix de la direction paraît connaître une évolution sensible, en intégrant une dynamique de négociation nouvelle sur le devenir des salariés dans et hors de l'entreprise à travers des 'accords de gestion prévisionnelle de l'emploi et des compétences' et des 'accords de méthode' (Jobert 2007). Comment analyser cette ouverture à une forme de négociation succédant à une pratique collective privilégiant jusqu'ici l'opposition à des projets de réorganisation en mettant en jeu ce qui était vu comme une prérogative centrale de la direction dans l'entreprise, l'emploi ? Si, dans des situations extrêmes et parfois très conflictuelles, la négociation tend à porter prioritairement sur les indemnités de départ des salariés, elle fait apparaître plus généralement un besoin nouveau de prendre en compte les attentes des salariés tant à l'égard de leur avenir personnel, qu'à celui de l'évolution de leur entreprise. En quoi cette démarche nouvelle qui tend à lier information et consultation des représentants des salariés d'une part, négociation avec les responsables syndicaux d'autre part, traduit-ellel'émergence d'un registre d'action collective se rapprochant, à l'échelle de l'entreprise, d'une « approche par les capacités »?

Pour analyser l'émergence de ce registre, nous proposons d'envisager la possibilité d'une «approche par les capacités », non plus de manière «théorique » comme principe de philosophie politique décrivant un mode possible de fonctionnement de régimes démocratiques, mais à partir d'un point de vue « empirique » ou encore, sociologique, comme une forme spécifique de mobilisation des acteurs. En effet, les cadres juridiques dans lesquels s'inscrivent les restructurations constituent les bases d'un ancrage, Sen parle d'une «positionnalité » et d'un «voisinage » (Sen 2010 p. 199 et s.), dans lequel s'exercent la rationalité et la réflexivité des individus. Il en résulte que l'«approche par les capacités » ne désigne plus simplement un principe général permettant à une «élite intellectuelle et administrative »,d'engager des débats sur la voie d'une «bonne gouvernance » au sein d'un «lieu neutre» afin de peser sur 1' «idéologie dominante» (Hauchecorne 2010). Elle correspond davantage àune forme de participation des individus concernés et de leurs représentants à un choix social situé, où les libertés de choix, indissociablementcollectif et individuel, des salariés, sont au cœur de débats conduisant à appréhender 1' «entreprise » comme une entité collective qui se dessine à partir des apports en capacité qu'organise le contrat de travail. Cela suppose d'intégrer dans la capacité de chaque individu concerné, une dimension de choix collectif que nous proposons de désigner comme une «capacité politique ». Comme mode d'exercice de cette «capacité politique », l' «approche par les capacités » fait une place à une réflexivité complexe liant pour chaque individu un retour sur 
lui-même et sur la collectivité auquel il appartient. En ce sens, le droit du travail comme base d'une « capacité politique » peut être qualifié de « réflexif » (Deakin et Koukiadaki 2012).

Pour envisager l' «approche par les capacités » comme registre de participation des salariés dans l'entreprise, nous envisagerons en premier lieu la manière dont Sen mobilise cette approche dans une perspective spécifique sur le développement où la démocratie et, plus généralement, la question des choix sociaux occupent une place centrale.Nous examinerons, dans un deuxième temps, les conditions d'une telle approche dans l'univers du travail. Nous tenterons, enfin, de préciser les traits de ce registre émergent à travers une série de monographies portant sur des situations de restructurations ${ }^{3}$.

\section{L' "approche par les capacités » : la démocratie au cour du développement}

L' «approche par les capacités » correspond à la constitution d'un ensemble d'informations permettant de rendre compte des capacités individuelles dans leurs multiples dimensions en vue d'éclairer les choix sociaux dans un régime démocratique. Elle implique une conception de la capacité individuelle comme liberté de choisir sa vie, tant à travers des choix individuels que par des choix collectifs. La démocratie est ainsi présentée par Sen comme un facteur essentiel du développement. Elle a été concomitante, en Inde, avec une disparition des famines que le pays a connues pendant la colonisation anglaise. Mais cette dimension primordiale de la démocratie dans le fonctionnement de «filets de sécurité » évitant les catastrophes humanitaires ne constitue pas sa seule vertu. Plus profondément, l'enjeu de la démocratie est celui de la participation des citoyens aux choix collectifs qui les concernent, par des services, des garanties et des droits définissant un spectre de libertés élémentaires à la base du processus de développement.

\subsection{Une certaine idée du développement}

Sen part d'une conception du développement qui, tant dans sa finalité que dans ses moyens, va au-delà de la vision en terme de «décollage» du PIB. La finalité du développement, pour lui, ne se définit pas simplement par la croissance de la valeur ajoutée, elle se définit plus largement par le progrès ${ }^{4}$ de la liberté des individus. La liberté individuelle

\footnotetext{
${ }^{3}$. Les analyses présentées se fondent sur un corpus de vingt cinq monographies réalisées en France dans une perspective comparative au sein des programmes européens EUROCAP (2003-2006) et CAPRIGHT (20072010), en vue de dégager les enjeux d'une « approche par les capacités » dans les situations de restructurations.

4. «L'idée de progrès social raisonné est essentielle à l'approche présentée dans ce livre » (Sen 2003a, p. 364).
} 
s'entend comme liberté de projet, liberté de faire, ou encore «liberté positive» (par opposition à la « liberté négative » définie par Berlin (1968) comme absence de contrainte). Cette «liberté positive » constitue la «capacité» d'un individu, c'est-à-dire sa «liberté de mener différentes sortes de vie, [qui] correspond exactement à l'ensemble formé par différentes combinaisons de fonctionnements humains, ensemble en lequel une personne est à même de choisir sa vie » (Sen 2003b, p. 64). Dans cette approche, les «fonctionnements » sont définis comme des «pouvoirs d'accomplir», parmi lesquels on compte des «fonctionnements élémentaires» comme pouvoir se nourrir ou se soigner, et des «fonctionnements [...] plus complexes, bien que leur valeur soit encore largement méconnue : par exemple, ressentir de l'estime de soi-même ou participer à la vie de sa communauté »(ibid.). Concevoir le développement comme développement de la liberté suppose alors de concevoir la liberté ou la «capacité » des individus, non à partir d'un ensemble de «fonctionnements » préalablement donnés, mais comme un ensemble ouvert à partir d'un certain nombre de libertés élémentaires garanties. Si l' «approche par les capacités » repose sur des fonctionnements primordiaux, elle ne se réduit pas à la « conversion » de ressources en vue d'accomplir des fonctionnements préalablement donnés. Elle intègre également l'exploration de fonctionnements nouveaux, en rupture avec la reproduction de pratiques traditionnelles. Développement, liberté et capacité sont donc étroitement liés, en remettant en cause le postulat d'une nature humaine, soit comme rationalité instrumentale, soit comme ensemble d'aspirations que les ressources issues du développement combleraient progressivement. En ce sens, « la Capability Approach est très savamment inachevée. » (De Munck 2008, p. 28).

Dans la mesure où la liberté, ou encore, la capacité des individus est l'objet même du développement, ce que Sen présente comme une «approche par les capacités » implique de partir du développement, pour analyser ensuite les capacités des individus. Ce lien entre développement et liberté individuelle évoque le lien établi par Durkheim entre sociologie et psychologie. La sociologie, comme analyse de la dynamique des sociétés où la division du travail joue un rôle clé, ne repose pas sur la connaissance d'une nature humaine préalablement donnée. A l'inverse, c'est elle qui apporte des éclairages sur la dynamique de la psychologie des individus : «L'homme est, pour nous, moins un point de départ qu'un point d'arrivée. Nous ne commençons pas par postuler une certaine conception de la nature humaine pour en déduire une sociologie; c'est plutôt à la sociologie que nous demandons une intelligence progressive de l'humanité. » (Durkheim 1975 [1909], p. 185). De manière analogue, dans le modèle de Sen, l'analyse du développement comme dynamique historique d'ensemble, 
contribue à apporter une «intelligence progressive» des capacités humaines. Le développement apparaît ainsi comme un processus dans lequel la garantie de libertés élémentaires constitue une base, pour un élargissement de la liberté sous l'impulsion des acteurs: «Les objectifs que les gens peuvent atteindre dépendent des possibilités économiques, des libertés politiques, de l'environnement social et des conditions qui favorisent l'accès à la santé et à l'éducation ou qui encouragent les initiatives. La codification institutionnelle de ces opportunités dépend, en retour, de la manière dont les gens exercent leurs libertés, par l'intermédiaire de leur participation aux choix sociaux et à l'élaboration des décisions publiques qui améliorent ces possibilités. » (Sen 2003a, p. 17).

\subsection{Cinq libertés élémentaires}

Le développement repose des formes élémentaires de la liberté individuelle, dont les différents aspects se renforcent mutuellement avec, comme horizon, la participation des individus aux différents choix collectifs qui engagent leur avenir.

\section{- 1. La démocratie.}

Au centre de cette liberté conçue dans un sens large, la démocratie comme capacité de choisir et critiquer les dirigeants, et de participer aux choix collectifs apparaît comme essentielle et se retrouve à maints égards dans les autres aspects de la liberté. La place primordiale que Sen confère à la démocratie s'inscrit dans la discipline économique que constitue la «théorie des choix sociaux» et repose sur la discussion du «théorème d'impossibilité » établi par Arrow dans les années 1950. Ce théorème reprend et développe le paradoxe de Condorcet, en montrant la contradiction potentielle entre préférences individuelles (conçues comme un ordre de choix entre l'ensemble des états sociaux envisageables) et hiérarchie des états sociaux établie par un vote majoritaire,concluant sur cette base à l'impossibilité d'un choix social démocratique. Mais la discussion de la portée de ce théorème a été centrale dans la réflexion qui s'est engagée autour de la théorie des « choix sociaux », comme Sen le souligne aujourd'hui (Sen 2011) après la conférence prononcée à la remise du Prix Nobel (Sen 1999). Pour Sen, en effet, le théorème d'impossibilité ne remet pas radicalement en cause la validité de choix collectifs démocratiques : «Ce théorème élégant est l'une des contributions analytiques les plus remarquables dans le champ des sciences sociales. Mais il n'invalide en rien les mécanismes de décision qui reposent sur des bases 
d'informations plus larges ou différentes de celles nécessaires aux règles de vote. » (Sen 2003a, p. 329).

Sans nier la portée de ce théorème, Sen en a montré à plusieurs reprises les limites en mettant l'accent sur les «bases informationnelles » à la base des choix sociaux justifiant une « approche par les capacités ». La justification de cette approche repose notamment sur le fait que la rationalité individuelle des agents intègre des dimensions qui ne peuvent être rapportées à une dimension unique telle quel'utilité des individus. Ainsi, aux Etats-Unis, l'attachement à un régime de liberté constitue un facteur explicatif dans les migrations qui ont conduit les esclaves affranchis à quitter les Etats du sud, en acceptant des niveaux de vie inférieurs. Les tentatives de les attirer dans des « formes diverses d'organisation du travail calquées sur le modèle servile, mais en échange cette fois, de rétributions élevées, ont toutes abouti à l'échec. » (Sen 2003a, p. 47). Sen avance un argument analogue pour expliquer l'effondrement des régimes communistes dont la faillite est incompréhensible « si l'on s'en tient aux données économiques brutes, qu'il s'agisse des revenus ou de l'espérance de vie. » (Sen 2003a, p. 156). Les régressions constatées parfois dans certains anciens pays communistes, comme pour l'espérance de vie en Russie, n'ont pas encouragé la revendication d'un retour à l'ordre ancien.

Face à la diversité des fins poursuivies par les individus, la démocratie va au-delà de la désignation de représentants par le vote et conduit à une pratique de délibération en mesure de confronter et de comparer les préférences et, plus largement, les points de vue des citoyens, variant en fonction des fins qu'ils ont des raisons de valoriser. Elle agit dans le sens d'une extension des «bases informationnelles » sur lesquelles se fondent les choix sociaux, à partir de 1' «approche par les capacités » qui se dégage au terme de délibérations permettant de saisir les problèmes à envisager, d'élaborer des solutions et d'établir des priorités. La démocratie est le fondement de «choix sociaux » qui se rapprochent de l'expression d'une «volonté générale » à partir de l'identification d'un «bien commun » distinct des intérêts individuels immédiats des acteurs, et qui conduisent à des résultats supérieurs pour les individus à ceux qu'ils obtiendraient par le jeu de stratégies individuelles guidées par leur seul intérêt ${ }^{5}$. Elle repose sur la capacité des individus à assumer réflexivement la position d'un «spectateur impartial », dans les choix sociaux auxquels ils sont amenés à prendre part.

5. En retrouvant un écho au commentaire du Contrat social présenté par Runciman et Sen (1965): « The conflict between the will of all and the general will arises [...] because of the difference between the outcome of individual strategy and of enforced collusion which arises under the conditions of non-cooperative, non-zero-sum game. » (Runciman et Sen, p. 557). 
Si la démocratie politique apparaît comme première dans les analyses de Sen, elle initie également un processus de démocratisation dans les groupes existant au sein d'une société. De ce point de vue, «The subject of Social Choice Theory is to investigate how a group of people who make up the collectivity, a nation, a community, a family, a committee, an academy or whatever, how they can aggregate or put together rationally, the diverse and possibly divergent preferences and priorities the different members of that group have. » (Sen 2009, p. 264). Le cas de la famille revient fréquemment dans les écrits de Sen, à travers l'évolution que suscite l'émancipation des femmes par l'éducation et la participation au marché du travail, en renforçant leur «capacité politique»dans les choix collectifs de ce groupe. Cela nous conduit à envisager également ce processus dans les milieux de travail, au sein desquels «le statut salarial reconnaît cette capacité politique, mais sous des formes limitées. » (Eymard-Duvernay 2008, p. 208).

\section{-2. Les «facilités économiques »}

Les «facilités économiques » correspondent aux « opportunités offertes aux individus d'utiliser les ressources économiques à des fins de consommation, de production ou d'échange. » (Sen 2003a, p. 59). Mais en concevant le développement comme développement des libertés individuelles, plutôt que de la valeur ajoutée, Sen suggère que les «facilités économiques » sont une finalité parmi d'autres dans cette dynamique. Ainsi, une «approche par les capacités » conduit à constater les effets négatifs de trop fortes inégalités dans ce domaine $^{6}$ en envisageant des situations de retrait, voire de servitudes, où les individus voient leur liberté de choix, tant individuels que collectifs, limitée. Elle suggère desmécanismes de redistribution sous la forme, par exemple, de réformes agraires ou de taxation des revenus les plus élevés.

\section{- 3. Les « opportunités sociales »}

Les « opportunités sociales » correspondent à la prise en charge des soins de santé et de l'éducation des enfants par des « services sociaux fonctionnels », facteurs importants pour garantir un accroissement de la «qualité de vie». Elles ont des effets économiques à travers la capacité accrue d'individus formés et en bonne santé, de participer non seulement aux activités économiques avec une efficacité accrue, mais aussi à la vie démocratique.

\footnotetext{
${ }^{6}$. Comme dans le cas de ces paysans indiens terrorisés par de grands propriétaires et réduits à une forme de servage.
} 
- 4. La «non-duplicité dans les échanges »

L'existence d'un Etat de droit, qui garantisse une transparence et une non-duplicité dans les échanges, constitue une autre condition pour le développement, non réductible aux précédentes. Elle suppose l'existence de juridictions non corrompues garantissant le «monopole de la violence physique légitime », en limitant le poids de la violence privée exercée par des mafias. En effet, pour Sen «Le fonctionnement du marché ne met pas seulement en œuvre des échanges " autorisés » [c'est-à-dire portant sur des objets licites], il dépend aussi de solides fondations institutionnelles (les dispositions légales qui protègent les droits des contrats) et d'une éthique de comportement (un contrat est reconnu comme un accord tacite, sans nécessité de procès permanents pour en faire respecter les termes). La confiance en la parole donnée est un ingrédient essentiel du marché » (op. cit., p. 344). Dans cette perspective, «tout code de conduite élémentaire régissant les relations d'affaires joue le même rôle que l'oxygène. » (op. cit., p. 345). Ces fondations institutionnelles deviennent si familières qu'elles ne se font sentir que quand elles viennent à manquer. Ainsi, « une réalité familière qui ne suscite ni émerveillement ni surprise à Londres, Zurich ou à Paris, peut être sujet d'insurmontables difficultés au Caire, à Bombay, à Lagos ou à Moscou, là où l'instauration des normes et des institutions d'une économie de marché fonctionnelle reste un défi incertain. » (op. cit., p. 346).

- 5. Les « filets de sécurité »

Enfin, la «sécurité protectrice » permet de préserver les plus vulnérables par des systèmes d'allocations et des fonds exceptionnels d'intervention (pour prévenir les famines, indemniser les chômeurs...). Cependant, on pourrait objecter que l'existence de ces filets peut avoir des effets pervers : le système de Speenhamland prévoyant, dans l'Angleterre de la fin du XVIIIe siècle, le versement de subsides publics aux travailleurs bénéficiant de revenus insuffisants est vu par Polanyi (1983) comme la source du « désarmement moral » de la classe ouvrière britannique, par rapport à l'engagement de la classe ouvrière française dans la Révolution. Actuellement, l'indemnisation du chômage dans les pays européens rend tolérable une situation de chômage élevé, sans écarter la question essentielle de l'emploi, compte tenu, selon Sen, des effets dévastateurs du chômage sur les capacités des individus et leur implication dans la vie démocratique.

De manière générale, la conception du développement comme développement de la liberté des individus permet de mettre en évidence les limites d'une analyse du 
développement focalisé sur la «compétitivité » immédiate des pays. Le développement apparaît comme inconcevable en prenant pour base la misère et l'avantage comparatif que constituerait l'existence d'une main d'œuvre docile et bon marché, mais dans un état de santé médiocre et peu formée et dans une situation où la domination des employeurs reposerait sur la dictature d'un pouvoir autoritaire. Ainsi, les analyses de Sen dessinent un processus où le développement repose sur des «libertés » élémentaires, permettant aux individus de se trouver en mesure de prendre aux choix collectifs qui renforceront, à leur tour, ces « libertés » et donc le développement.

\subsection{Le développement comme processus}

Cette conception de la liberté comme moyen et comme fin $\mathrm{du}$ développement,constitue le fondement d'une démarche analytique permettant de mettre en lumière un mécanisme de développementqui va au-delà d'une conception «culturaliste ». Le développement ne repose pas sur l'existence de traits culturels et de traditions créant des conditions plus ou moins favorables à son émergence, bien au contraire, il implique une remise en cause des sujétions traditionnelles qui entravent les individus. Cela se manifeste particulièrement à travers l'éducation qui, pour les femmes, se révèle être un facteur d'émancipation encourageant à son tour la participation de ces dernières à la vie démocratique: «Si Condorcet, par exemple, souligne l'importance de l'éducation des femmes, c'est, entre autres, parce qu'il comprend qu'on a besoin que les femmes fassent entendre leur voix dans les affaires publiques comme dans la famille et la vie sociale. Et, lorsqu'elles le feront, ces voix féminines pourront, à leur tour, nous amener à donner priorité dans l'action publique à l'éducation des femmes, dans le cadre de la promotion de la justice dans la société, tant pour ses bénéfices directs que pour ses effets indirects. » (Sen 2010, p. 148).

De manière analogue, la liberté du travail comme «libre participation au marché du travail » ne se réduit pas à la seule domination du travailleur par l'employeur. Elle apparaît comme un levier essentiel pour remettre en cause des formes d'appartenance sociale qui poussent les individus à mettre entre parenthèses leurs aspirations, pour s'inscrire dans le maintien de l'ordre communautaire'.

7. Ce processus se rapproche de la remise en cause de la société traditionnelle que décrit Pierre Bourdieu à travers la confrontation entre cette société et la société coloniale capitaliste : «Il est vrai que rien n'est plus étranger à l'économie précapitaliste que la représentation du futur comme champ de possibles qu'il appartient au calcul d'explorer et de maîtriser » (Bourdieu 1977, p. 19). La question des choix individuels, essentielle dans le 
Cette remise en cause prend parfois la forme d'une rupture violente, comme dans le cas de la Guerre de Sécession aux Etats-Unis aboutissant à l'abolition de l'esclavage, ou dans celui de l'abolition des privilèges et des corporations au cours de la Révolution Française. Mais elle se prolonge sous la forme d'un processus plus continu, dans le cas où les oppressions subies et les appartenances communautaires sont si fortes que les individus « apprennent à contrôler leur plaisir et leurs désirs en conséquence, puisque cela n’a guère de sens d'aspirer à des choses inaccessibles. » (Sen 2003a, p. 106). La croissance de l'activité professionnelle des femmes dans les pays en développement illustre ce processus, avec une amélioration paradoxale de l'état de santé des femmes actives et de leurs enfants. Cette situation tient, selon Sen, à l'affirmation d'une individualité et d'un souci de soi face aux attentes infinies auxquelles les femmes ont à faire face dans la communauté familiale. Comme dans le cas de l'éducation, elle tient également à la participation aux choix collectifs familiaux, en permettant aux femmes actives de peser sur la vie du foyer par les revenus qu'elles apportent, ou encore par le besoin de concilier leurs activités dans la famille avec leurs engagements professionnels en contrôlant, notamment, leur fécondité. On voit ainsi se dessiner un processus dans lequel le principe d'égale liberté dans la participation au marché du travail tend à se renforcer à la fois dans les rapports entre travail et hors-travail, ainsi que dans le travail lui-même. Le processus est cependant loin d'être terminé dans les pays dits « développés » et «L’affirmation juridique du principe de l'égalité précède ici sa réalisation de fait. Le droit se présente là comme un projet [...]. Mais [...] seule la connaissance des rapports sociaux effectifs permet la reconnaissance du décalage entre la réalité effective et la norme que le droit énonce, et la définition des voies et des moyens par lesquels le droit peut devenir efficace. » (Laufer 2003, p. 169).

\subsection{La visée sociologique de l'« approche par les capacités»}

La question du développement est donc au centre des analyses économiques proposées par A. Sen, avec l'ambition de proposer un «nouveau modèle de développement » dans lequel la liberté des individus se présente comme la valeur primordiale. En ce sens, ces

développement comme développement en liberté, se trouve ainsi posée au travers du contraste entre « l'entraide » communautaire et la coopération contractuelle : «Rien ne s'oppose plus radicalement à l'entraide, qui associe toujours des individus unis par des liens de consanguinité réelle ou fictive, que la coopération qui mobilise des individus sélectionnés en fonction des fins calculées d'une entreprise spécifique : dans un cas, le groupe préexiste et survit à l'accomplissement en commun d'une œuvre commune ; dans l'autre cas, trouvant sa raison d'être hors de lui-même, dans l'objectif futur défini par le contrat, il cesse d'exister en même temps que le contrat qui le fonde. » (op. cit., p. 26). 
analyses se caractérisent par une portée normative importante, conduisant à des propositions en termes de réduction des inégalités et, plus profondément des injustices, mettant en cause le un modèle de développement en termes d'accroissement de la liberté des individus. Mais cette dimension normative prend une forme spécifique dans le domaine de la théorie économique. Elle se traduit par une distance à l'égard de ce que Sen définit comme un « institutionnalisme transcendantal », c'est-à-dire une démarche déduisant a priori les institutions parfaites en vue de la recherche d'un optimum social à partir de la conception préalable d'une «nature humaine » prise dans la tension entre égalité et utilité. Elle prend la forme d'une recherche d'amélioration à partir des injustices constatées dans un «état social » donné, mais non prédictible compte tenu à la fois de la manière dont les individus exercent leur liberté et à l'inverse de situations où ils sont conduits à «faire de nécessité vertu », en se plaçant d'euxmêmes en dehors d'un débat collectif dont ils jugent déraisonnable d'attendre quelque chose.

De ce point de vue, la démarche fait une place à une visée sociologique à au moins trois titres.

1. Elle repose sur une révision du modèle de rationalité des agents de la théorie économique, en partant d'une remise en cause du primat de la «rationalité instrumentale » telle qu'on la trouve dans l'hypothèse de préférences individuelles formulées à partir d'un programme de maximisation de leur utilité par les agents. Elle trouve ainsi un écho dans une démarche de «sociologie analytique » telle que la développe Raymond Boudon, au travers de la notion de «rationalité axiologique » intégrant la dimension des valeurs dans les motifs d'action des agents (Boudon 1999). Si la liberté est, pour Sen, la finalité du développement, c'est qu'elle est également un motif d'action des agents induisant un processus de rationalisation complexe évoquant la conception wébérienne dans laquelle, à la rationalisation économique et institutionnelle, répond une «rationalisation diffuse» (durchrationalisierung) des croyances et des valeurs des agents. La démocratie et l'Etat de droit constituent ainsi une réalisation observable de ce processus, dont le caractère fragile mais irréversible tient selon Boudon au poids qu'ils ont pris dans les motifs individuels.

2. La démarche suppose, de plus, une observation des réalités sociales destinée à établir l'état de la société, dans le contexte de débats sur la part d'injustice qu'il revient à l'Etat de combattre. On retrouve sur ce point l'importance de la «statistique », comme activité publique d'observation de la société en vue d'alimenter les bases 
d'information du pouvoir politique (Desrosières 1993$)^{8}$ et la critique des indicateurs économiques européens développée par Salais (2008). Cette pratique d'observation,qui va au-delà de la stricte démarche quantitative,est au centre de l' «approche par les capacités », définie comme « une méthode d'ordre général qui oriente l'attention vers l'information sur les avantages individuels, jugés en termes de possibilités et non en fonction d'un «projet» spécifique sur la bonne façon d'organiser la société »(Sen 2010, p. 285). Elle implique une attention aux agents eux-mêmes dans la mesure où «la perspective des capacités se soucie inévitablement d'une pluralité d'aspects de nos vies et de nos préoccupations [...] et propose d'abandonner la focalisation sur les moyens d'existence pour s'intéresser aux possibilités réelles de vivre »(op. cit., p. 286). A ce titre, elle justifie également une pratique d'enquête qualitative partant de la vie des agents, en vue de saisir la manière dont ils «convertissent» les ressources à leur disposition pour développer leur capacité (Zimmermann 2008).

3. La focalisation sur les « capacités » des individus est corrélée à une conception de la démocratie tirée de la discussion de la théorie des choix sociaux et, plus particulièrement, du théorème d'impossibilité de K. Arrow. Dans cette perspective, la démocratie ne se réduit pas à une procédure de vote mais repose sur les «bases informationnelle» qui se dessinent dans une libre discussion. Il en résulte une attention pour les formes de délibération qui se font jour dans la vie sociale, à la fois au niveau de l'Etat, à celui de la famille (dans le cas du développement l'activité professionnelle féminine) et plus globalement à celui de toutes les formes de « comités » ${ }^{9}$ en charge de la prise de décision engageant l'avenir de groupes humains. Le «comité » se distingue du «groupe de pression », dans la mesure où son objectif n'est pas la simple défense d'un intérêt particulier, par exemple celui d'une profession ou d'une classe sociale contre le reste de la société, mais l'identification d'un intérêt général dans un groupe donné, constitué d'individus différents et capables de dégager la position d'un «spectateur impartial ${ }^{10}$. Cette analyse de la démocratie comme

8. «Même les inventeurs de l'estimation quantitative du revenu national, qui a fait l'objet de tant d'attention et d'adhésion, ont tenté d'expliquer que leur centre d'intérêt ultime était la richesse des existences humaines, bien que ce soient leurs indices et non leurs motivations qui ont largement marqué les esprits. » (Idée de Justice, p. 278).

9. Sen (1999) évoque notamment les commissions parlementaires, les conseils de famille et les comités de sélection universitaire.

${ }^{10}$. On retrouve une réflexion proche de celle de Boudon (2006). 
organisation d'une « réflexion collective » au sein de « conseils » trouve selon nous un écho dans la conception de la démocratie présentée par Durkheim (1950).

Dans cette perspective, l' «approche par les capacités » induit une construction en « abyme » dans laquelle une enquête sur la capacité des agents implique à la fois un regard sur leurs choix personnels, mais aussi sur leur participation à des procédures de choix collectifs. Dès lors, ils sont en position eux-mêmes de mettre en œuvre une «approche par les capacités » pour constituer les bases d'informations visant à éclairer les choix collectifs dans lesquels ils se trouvent engagés. Cela permet de sortir d'une approche normative visant à évaluer directement la portée de l'action publique sous l'angle de l'espace des «fonctionnements individuels» dans lequel les individus choisiraient individuellement leur vie, pour concevoir une sociologie positive de l' «approche par les capacités ». Ainsi, « le retour à une démarche de socio-économie positive permet de donner une extension considérable aux opérations d'évaluation, au cœur de l'analyse de Sen : réservées[par Sen] aux évaluateurs surplombant l'espace économique, qu'ils soient des politiques ou des chercheurs, elles innervent [en fait] l'ensemble des activités »(Eymard-Duvernay 2008, p. 209).

\section{2. $L^{\prime}$ "approche par les capacités » appliquée au travail}

Dans les analyses de Sen, la « liberté de participer au marché du travail » repose sur un état de droit visant à prohiber les formes de travail contraint à travers, principalement, l'institution du contrat de travail. Mais si le contrat établit une liberté individuelle -relativede choix du travail, il ouvre également au travailleur l'accès à une activité, la plupart du temps collective, qui oblige à s'interroger sur la collectivité qui se dessine ainsi. Cette collectivité présente des caractéristiques spécifiques à l'égard des communautés traditionnelles auxquels appartient l'individu. Elle repose sur un choix individuel du salarié et de l'employeur et peut être remise en cause unilatéralement par chacune des deux parties au contrat. Elle présente néanmoins des traits communs à tout groupement, en posant la question de la participation de ses membres aux choix collectifs qui engagent son avenir et celui de ses membres.

\subsection{Le contrat de travail comme " apport en capacité »}

Pour Sen, la «liberté de participer au marché du travail » est ancrée dans la «liberté de participer au marché » qui repose, de manière générale, sur la liberté contractuelle. Mais le 
contrat de travail se caractérise par l'existence d'une situation de «subordination juridique » qui se manifeste par le pouvoir de directive et de contrôle de l'employeur ou par l'exercice du travail dans un local de l'entreprise ou par l'appartenance à un « service organisé » par l'employeur. Si la «liberté de participer au marché du travail » peut conduire, par exemple pour les femmes, à une forme d'indépendance à l'égard du groupe familial, elle est donc porteuse d'une menace substantielle sur la liberté du travailleur.

Cependant, le contrat de travail ne se réduit pas au rapport entre un employeur et un travailleur, c'est-à-dire à la transaction entre un «salaire » et un « emploi ». Il se définit par un « dualisme structurel » impliquant d'envisager, en dehors du rapport travailleur-emploi, les « relations professionnelles » qui se nouent par l'entrée du salarié dans la collectivité dessinée par la division du travail au sein de l'entreprise (Jeammaud 1989). Cette entrée dans une collectivité se manifeste sur le lieu de travail dans la mesure où, «de tous les facteurs de production, et de façon contrastée au regard du capitalisme financier ou de la technologie, le labour power est une force qui s'exerce - et ne peut s'exercer, d'ailleurs - que sur le shopfloor. » (Bélanger et Thuderoz, 2010, p. 430). Au-delà de l'atelier, même si l'atelier et les enquêtes sur les motivations individuelles des ouvriers ont constitué un terrain fondateur pour la sociologie du travail (Borzeix et Rot 2010), l'espace du travail vécu se dessine à partir des relations qu'entretiennent les membres d'un atelier et d'un service avec des travailleurs appartenant à d'autres ateliers et à d'autres services, voire à d'autres établissements, comme dans le cas des ingénieurs en charge du développement de nouveaux produits ou de nouveaux procédés de production dans des établissements de prototypage et de production. Elle paraît davantage liée à la détermination d'un ensemble productif cohérent, pour lequel il est convenu de parler d' «entreprise ». En ce sens, le contrat de travail définit les formes prises par l'apport en capacité des travailleurs constitutives, à certains égards, de l'entreprise.

L'existence de cette collectivité «vécue » et instituée par le droit du travail qui se greffe sur le contrat de travail, implique d'envisager la « capacité politique » des salariés dans l'espace de l'entreprise. Il en résulte que l'entreprise n'est pas seulement « capacitante » dans le sens du développement des savoir-faire individuels des salariés par un employeur plus ou moins ouvert aux projets des salariés concernés, et lié par l'obligation d'adaptation des salariés à leur poste de travail résultant du principe de la bonne foi dans l'exécution du contrat ${ }^{11}$. En

11. Sous cet angle, il est possible d'identifier de «bonnes pratiques » dans les politiques de ressources humaines, comme dans le cas de Bigtruck décrit par Zimmermann (2011), où même les syndicalistes disent être «membres de longue date de la «famille Bigtruck» (Zimmermann 2010 p. 169). Mais en se focalisant sur la politique des employeurs, cette démarche repose sur une forme de 'paternalisme méthodologique' en concevant 
amont de cette politique managériale, l'entreprise est elle-même constituée par les apports en capacité qu'organise le contrat de travail, à travers les coordinations qui se font jour dans le travail comme activité collective, et la participation des salariés aux choix sociaux qui engagent leur avenir et celui de leur entreprise. Cette «capacité politique » comme pouvoir de peser sur les choix engageant l'avenir de l'entreprise et de ses salariés, ne se réduit pas aux trois possibilités que constituent exit, voice and loyalty dans l'analyse d'Albert O. Hirshman. Elle se définit ainsi moins comme une «capability for voice » (Bonvin 2008) se manifestant ponctuellement face à des décisions jugées comme inacceptable, que comme une capacité d'évaluation sur l'ensemble des décisions concernant les salariés, non exclusive des modalités exit $^{12}$, voice ${ }^{13}$ et loyalty ${ }^{14}$.Cette évaluation s'opère non seulement en regard de l'intérêt individuel des salariés, mais aussi en regard de leur connaissance empirique du travail et de leur perception de l'entreprise comme collectivité.

\subsection{L' «approche par les capacités » comme registre des relations professionnelles}

L' «approche par les capacités » se présente dans l'œuvre de Sen comme un principe d'évaluation du caractère plus ou moins démocratique des choix collectifs au niveau, principalement, de l'action étatique. De ce point de vue, elle a une portée critique forte à l'égard d'autres modalités de choix collectifs en dévoilant la limitation de leur caractère démocratique. Elle suggère de revenir sur ces différentes modalités possibles, en se focalisant en premier lieu sur la modalité la plus opposée à la démocratie, la dictature, selon le partage démocratie-dictature au centre du théorème d'impossibilité d'Arrow. Mais la dictature comme pouvoir d'un individu, ne constitue que l'une des modalités possibles de l'atteinte à la démocratie. Une autre modalité possible est celle du gouvernement fondé sur des « indicateurs clés en main » qui correspond à un affaiblissement de l'arbitraire, mais repose sur un verrouillage des «bases informationnelles» de choix collectif résultant de leur monopolisation par des experts. Une dernière modalité nous semble renvoyer à ce que Sen

les capacités des salariés comme le résultat des initiatives managériales dans le domaine de la formation professionnelle.

12. Comme nous le verrons à travers le souci de ménager, en cas de restructuration, des conditions permettant aux salariés de « réaliser leur projet » en dehors de l'entreprise.

${ }^{13}$. Par les grèves et les manifestations visant à attirer l'attention de l' «opinion publique », comme dans des affaires récentes avec les actions des salarié(e)s de Seafrance ou de Lejaby.

${ }^{14}$. Cette notion présente selon nous l'inconvénient de confondre les situations dans lesquelles la conformité du comportement des agents relève de la « routine », ou de la crainte à l'égard de l'autorité, et celles dans lesquelles elle se fonde sur une identification de « bonnes raisons » d'accepter les décisions managériales. 
présente comme «l'agitation ${ }^{15}$ comme recours de ceux qui restent à l'écart des choix collectifs les concernant, mais commencent à expérimenter le poids de leur action par des succès plus ou moins limités ${ }^{16}$. Etant établi que le choix social ne se concentre pas au niveau du pouvoir politique, mais se retrouve dans les groupes sociaux auxquels appartiennent les individus, il reste à voir dans le domaine du travail, et des relations professionnelles que dessine sa dimension collective, il est possible de retrouver de telles modalités du choix social.

Le droit du travail, en France, ouvre au salarié la possibilité de participer à deux grands types d'organisations collectives, le syndicat professionnel et le comité d'entreprise. Si la dimension 'entreprise' est immédiatement présente dans le comité d'entreprise, cela est moins le cas pour le syndicat professionnel que la loi tend à définir davantage par le «métier» et la «profession » pour établir la liberté de leur constitution même si, au-delà des «personnes exerçant un métier similaire », la loi évoque des personnes «exerçant des métiers connexes, concourant à l'établissement de produits déterminés »(L. 2131-2 CT). Ces deux organisations se définissent également par des fonctions différentes, «les organisations de salariés constituées en syndicats professionnels sont seules admises à négocier les conventions collectives et accords collectifs de travail»(L2132-2 CT), «le comité d'entreprise a pour objet d'assurer une expression collective des salariés permettant la prise en compte permanente de leurs intérêts dans les décisions relatives à la gestion et à l'évolution économique et financière de l'entreprise, à la formation professionnelle et aux techniques de production. »(L2323-1 al. 1).

Mais, en dépit de ces différences, la dimension 'entreprise' tend à prendre une place de plus en plus importante dans le fait syndical. La section syndicale d'entreprise reconnue par le législateur en 1968 définit les conditions de l'exercice du droit syndical dans l'entreprise. L'institution de négociations obligatoires annuelles sur les salaires par les lois Auroux de 1983 renforce le poids de l'entreprise dans la pratique syndicale. Le législateur a également établi un lien fort entre syndicat et comité d'entreprise en conférant aux syndicats le soin de négocier les conditions d'élection du comité, en leur attribuant l'exclusivité des listes

15. «Globalement, les possibilités qu'offre l'agitation publique sur les problèmes d'inégalités et de privations sociales commencent à être plus utilisées que par le passé [...]. Il y a eu dans ces derniers temps beaucoup plus d'actions dans le cadre de mouvements organisés souvent fondés sur des droits humains, tels le droit à l'éducation, le droit à la nourriture (au déjeuner dans les écoles), le droit aux soins médicaux de base, les garanties de protection de l'environnement et le droit à la « garantie de l'emploi ». » (Sen 2010, p. 418).

${ }^{16}$. Sen évoque ainsi le fait que des paysans indiens se «politisent» ou des « mouvements de femmes », selon un processus croissant, en sortant d'une situation où ils avaient appris « à adapter leurs plaisirs et leurs désirs en conséquence, puisque cela n'a guère de sens d'aspirer à des choses inaccessibles, choses dont ils n'ont d'ailleurs pas lieu, le plus souvent, d'imaginer l'éventualité. » (Sen 2003b, p. 57). 
présentées au premier tour. La reconnaissance d' «accords de méthode » destinés à aménager la procédure d'information et de consultation du comité d'entreprise et à définir les grandes orientations du «plan de sauvegarde de l'emploi » dans les procédures de licenciement collectif, par des lois de 2003 et 2005, a contribué à rapprocher encore les fonctions de négociation dévolues au syndicat et de consultation dévolues au comité d'entreprise (Jobert 2007). Enfin, la loi du 20 août 2008 a redéfini la représentativité des syndicats en la fondant sur le résultat des listes présentées aux élections des comités d'entreprise.

Ce contexte institutionnel influe sur les modalités de participation des représentants des salariés aux choix collectifs engageant l'avenir de leur entreprise, de leurs conditions de travail et de leurs emplois. Certes, il n'exclut pas un registre de confrontation, visant, par le recours à l' «agitation » sous la forme de grèves ou de manifestations, ou encore par le recours à la justice, à trouver un point d'équilibre avec un ou des employeurs, ou du moins à bloquer les décisions de ces derniers. Cependant, dans une situation de chômage élevé et de faible création d'emplois, il rend plus prégnant la question de l'avenir de l'entreprise et de celui de ses salariés. Face aux opérations de restructurations, ce contexte institutionnel oriente les acteurs vers une analyse des justifications des projets avancés par les directions d'entreprise selon une optique de «critique» (Boltanski 2010), et la formulation de propositions intégrant les attentes des salariés tant pour leur propre avenir que pour celui de l'entreprise. Une évolution se dessine alors entre ce que nous identifions comme deux registres d'action collective :

Le registre de la critique des justifications avancées par les directions se fonde sur une attention aux documents fournis par celles-ci et au respect des procédures d'information-consultation des institutions représentatives du personnel. Il se fonde sur la possibilité de saisir la justice et d'obtenir une suspension, voire une annulation des décisions et des éventuelles procédures de licenciement collectifs, en vue de bloquer les projets des directions. Il aboutit à ce qui apparaît fréquemment comme un «sursis » pour l'emploi, sans apporter d'éclairage sur l'avenir de l'entreprise et celui de ses salariés.

Le registre encore émergent de l' «approche par les capacités », visant une identification des problèmes posés par la décision managériale de restructuration et la recherche de solutionen liant une démarche d'observation des réalités de l'entreprise à la négociation d'accords collectifs. L'émergence de ce registre dans les restructurations que nous avons observées en France fait écho à l'orientation « réflexive » d'une législation européenne associant 
dans les licenciements collectifs information, consultation et négociation qui se retrouve dans d'autres pays européens, en particulier la Grande-Bretagne (Deakin et Koukiadaki 2012). Elle se manifeste par une logique de « projets », où la réflexion sur les « projets personnels » des salariés se trouve liée à celle sur l' « entreprise » comme projet collectif.

\section{Une « approche par les capacités » en situation de restructuration}

Les opérations qualifiées de «restructurations » désignent dans le Code du travail un ensemble de décisions qui se caractérisent par un effet commun, la «compression des effectifs $»^{17}$. Au travers des multiples décisions managériales que recoupe le terme, c'est donc l'emploi dans l'entreprise qui se trouve mis en cause. Ces décisions sont susceptibles de cristalliser de fortes oppositions autour de questions telles que le volume d'emplois supprimé, ou encore les conditions financières de départ des salariés à l'occasion des suppressions de postes envisagées. Les organes de représentation des salariés peuvent devenir des leviers importants de mobilisation, soit dans le sens d'un blocage de la décision supposant pour les représentants du personnel la possibilité de peser sur le centre de décision et pour l'entreprise de supporter le statu quo, soit dans celui d'une négociation indemnitaire dans des situations dominées par l'urgence que font peser une situation plus générale de crise ou par l'intransigeance de direction d'entreprises dans lesquelles le centre de décision est difficile à identifier. Si la grève a une portée limitée, dans le cas de fermeture de sites par exemple, la mobilisation collective sous la forme de manifestations et d'actions d'éclat susceptibles d'attirer l'attention de l'opinion publique et des élus locaux sont des ressources couramment utilisées dans ce type de situations (Adam et Reynaud 1978) en vue de dégager un soutien politique à la cause des salariés.

De telles opérations affectent durablement la vie de l'entreprise et de ses salariés, tant pour ceux qui partent que pour ceux qui restent. Elles impliquent non seulement une décroissance de l'emploi dans son volume, mais également une transformation du travail selon des processus de réorganisation dont la portée est large, en termes de répartition des tâches, de transformation de son contenu, de reclassement des salariés partant. Leur portée ne se réduit donc pas à une décision instantanée, mais suppose une préparation de la part des entreprises à travers notamment le concours de consultants, qui donne fréquemment l'alerte

\footnotetext{
${ }^{17}$. Cf. par exemple, dans le chapitre définissant les «attributions économiques » du comité d'entreprise, ses attributions concernant «l'organisation et la marche de l'entreprise » dans le cas spécifique des « restructurations et compressions d'effectifs » (art. 2323-8/art. 2323-11).
} 
aux élus du personnel et aux délégués syndicaux sur l'imminence d'une compression d'effectifs et appelle un regard sur la vie des salariés et le devenir de l'entreprise. Cela se retrouve dans le développement du droit du travail qui en France et en Europe, depuis les premières grandes restructurations des années 1960, a fait une place croissante à une obligation d'information et de consultation des comités d'entreprise. Les évolutions du cadre juridique développent ainsi les «chances » d'une discussion susceptible, au-delà du chiffrage de telles opérations, de prendre en compte le devenir collectif et individuel des salariés à partir d'une connaissance de leur travail et de leurs projets, c'est-à-dire d'une « approche par les capacités » des travailleurs dans l'entreprise.

\subsection{Ouverture d'un espace de débat sur l'emploi, la force de la critique}

Au cours des années 1960, dans le contexte de la construction de «champions nationaux », l'ouverture d'un débat dans l'entreprise semble avoir pris de court, dans un premier temps, un mouvement syndical organisé autour de la négociation salariale.En 1963, par exemple, les salariés de Neyrpic et leurs représentants se mobilisent en priorité pour la défense d'une échelle mobile particulièrement intéressante alors que son repreneur, Alstom engageait un processus continu de suppressions d'emploi (Béthoux, Brouté et Didry 2005). La stabilisation d'un cadre juridique établissant un «nouveau régime du licenciement économique », à partir de 1986, a néanmoins été la base d'un apprentissage institutionnel dans lequel la lutte pour l'emploi a rencontré quelques succès. Dans ce contexte, le fondement de l'action des comités d'entreprise a été une critique portant à la fois sur les projets et les données transmises par les directions, ainsi que sur la conduite des procédures d'informationconsultation, en vue d'obtenir, devant la justice, la suspension, puis l'annulation des procédures irrégulières. Un cadre juridique privilégiant une démarche d'information et de consultation et donc de débat sur l'entreprise, a été la base d'une conflictualité nouvelle autour de la «cause de l'emploi » en vue de bloquer les décisions managériales ou du moins de limiter l'ampleur des suppressions d'emplois (Didry 1998).

Cette démarche a eu une portée européenne à travers une décision judiciaire obtenue en 1997 par le comité d'entreprise européen de Renault retardant de plusieurs mois la fermeture de l'usine de Vilvorde dans la banlieue de Bruxelles, c'est-à-dire aux portes de la Commission Européenne (Didry 2001). Cette affaire a ouvert la voie à de nouvelles actions dans le cas du projet de fermeture de l'usine Opel d'Anvers par General Motors Europe, où l'action du comité d'entreprise européen dominé par IG Metall a permis d'éviter la fermeture 
du site (Da Costa et Rehfeldt 2010). Une démarche similaire se retrouve, en Belgique, dans le cas de l'usine Volkswagen de Forest, avec une action décisive du comité mondial du groupe allemand pour l'activité de l'établissement par la production d'un nouveau modèle.Cette démarche traduit une forme de délibération «par procuration », les salariés belges bénéficiant là aussi de l'autorité d'IG Metall pour éviter la fermeture du site (De Munck, Ferreras et Wernerus, 2010).

Ainsi, l'existence de procédures d'information-consultation dans les situations de restructuration a permis le développement de formes nouvelles de mobilisation collective, dans lesquelles le droit apparaît comme une « ressource » devant la justice, en vue de mettre en échec les projets des directions. Dans la perspective de Sen, cesmobilisations collectives indiquent une forme de choix collectif où prévaut une logique d'équilibre entre des forces, à partir duquel se déterminent le volume d'emplois sauvés et le niveau des indemnités versées aux salariés licenciés ou celui des préretraites pour les plus âgés. Mais ellestiennent pour acquis un attachement unanime des salariés à l'entreprise, justifiant une défense de l'emploi en tant que volume, etconduisant parfois à un décalage entre la stratégie des représentants du personnel et les attentes de certains salariés pouvant bénéficier, par exemple, de dispositifs de préretraite.

Cette stratégie connaît un développement important au cours des années 1990, sur la base d'une loi de janvier 1993 qui prévoit la possibilité pour les acteurs, comité d'entreprise, syndicats et salariés, de demander la nullité de la procédure de licenciement et de ses effets, donc les licenciements qui s'ensuivent éventuellement. Elle suppose une forte implantation syndicale, un comité d'entreprise ayant une connaissance approfondie de la gestion de l'entreprise et une mobilisation collective des salariés suffisamment large pour impressionner les directions. Elle est à l'origine d'une jurisprudence importante moins par le volume de décisions judiciaires, que par leur portée sur les différents aspects du licenciement collectif : contenu du plan social, régularité de la procédure de consultation, pertinence des documents économiques transmis par les directions. Les arrêts de la Cour de cassation dits «Samaritaine », en 1997, concluent ce processus en tranchant la question de savoir si la nullité de la procédure de licenciement implique la nullité de licenciements éventuels. Les actions de salariés de La Samaritaine, licenciés après une première reconnaissance de la nullité de la procédure de licenciement à la demande du comité d'entreprise, en 1995, donnent en effet lieu à une série d'arrêts de la Cour de cassation confirmant la nullité de leur licenciement et constatant donc leur réintégration. 
Cette jurisprudence est restée constante jusqu'aujourd'hui, suscitant, côté entreprises, des démarches d'évitement du licenciement collectif pour engager des processus plus continus de suppressionsd'emploi qui se traduisent par une montée des licenciements individuels et une diminution des licenciements collectifs (Bessy 2008). Elle a cependant contribué à préciser le contexte institutionnel, en conférant aux représentants des salariés un levier pour exercer une pression à la baisse sur les suppressions d'emplois et une pression à la hausse sur les incitations financières au départ des salariés.

\subsection{Desserrer la contrainte du départ par le «volontariat»}

Cette démarche,fondée sur une capacité critique à l'égard des projets des directions et de leur mise en œuvre, fait cependant apparaître plusieurs limites aux yeux des représentants des salariés. En premier lieu, elle permet de bloquer les décisions des directions, en allant parfois jusqu'à contester leurs justifications économiques, mais sans pouvoir engager de débats sur la stratégie économique de l'entreprise. En deuxième lieu, elle conduit ces représentants à perdre tout contact avec les salariés qui sortent du périmètre de l'entreprise, par mise en préretraite, licenciement ou externalisation. Dans le cas de la suppression d'emplois liée à la situation de quasi-cessation de paiement du groupe Alstom, en 2003-2004, la stratégie de confrontation choisie par les salariés de Belfort se heurtent à la faible mobilisation des salariés de la sous-traitance qui s'est constituée par un processus d'externalisation pendant la décennie précédente (Lefresne et Sauviat 2010). En troisième lieu, elle ne permet pas de prévenir, au terme d'une démarche d'anticipation, de nouvelles restructurations et suppressions d'emplois.

Les limites de cette démarche expliquent pour partie une évolution des politiques syndicales à partir d'une réflexion spécifique sur la 'flexicurité' encouragée à différents niveaux (européen et national), par les politiques de l'emploi dans un contexte de chômage élevé. Le point de départ est celui des obstacles que rencontre l'organisation de 'transitions professionnelles', dans une situation où un niveau de chômage élevé conduit les salariés sous CDI à se maintenir dans leur emploi face au risque de ne pouvoir en retrouver un à des conditions au moins équivalentes (Korver et Schmitt 2012). Les politiques syndicales visant une «sécurisation des parcours professionnels» (CFDT) ou une «sécurité sociale professionnelle » (CGT) entendent construire les bases de parcours professionnels qui ouvrent aux salariés la possibilité d'une mobilité professionnelle sans passer par l'état de chômeurs. Elles rompent avec une défense systématique de l'emploi, pour envisager la liberté du salarié 
dans son travail, liberté de partir ou de rester, qui trouve un écho dans un accord récent conclu au niveau du Dialogue Social Européen (Koster 2010).

Cette orientation syndicale trouve un écho dans les pratiques nouvelles des représentants du personnel en situation de restructuration, avec l'objectif de créer une forme de «volontariat » au départ la plus ouverte possible. Ces pratiques dessinent une première dimension de l' «approche par les capacités », en poussant les représentants des salariés à une discussion sur les projets des salariés et leur volonté de quitter l'entreprise pour leur réalisation. Elles se retrouvent ainsi en 2005 dans le cas de la fusion de deux entreprises d'édition phonographique, conduisantles représentants des salariés à négocier les conditions financières de départ dans un premier temps, pour ouvrir ensuite les débats sur la restructuration et l'évolution du travail proprement dites en relation avec le CHSCT (Didry et Jobert 2008). Dans ce cas, les conditions négociées suscitent un afflux de candidats au départ largement supérieur à celui des postes que la direction envisageaient de supprimer, nécessitant pour la DRH la mise en œuvre d'une deuxième procédure de licenciement collectif avec pour objectif, cette fois, de limiter le désir de départ des salariés. La fermeture de La Samaritaine en 2005 apparaît comme un lieu d'expérimentation pour la « sécurité sociale professionnelle » que promeut alors la CGT, avec la négociation d'un accord établissant le principe d'une poursuite des contrats de travail au cours de la recherche d'un reclassement dans le groupe LVMH, d'un autre emploi ou dans l'attente de l'accès à un régime de préretraite (op. cit.). Cette orientation se retrouve au sein des licenciements collectifs engagés au journal Le Monde en 2005 (Dupuy 2011), ou d'Hachette Filipacchi Associés (HFA) au sein du groupe Lagardère, avec, dans ce cas, la négociation d'un accord portant sur un volume trois fois plus large de départs que celui de la suppression d'emplois envisagés par la direction (Renoux 2010).

Ce renouvellement des pratiques repose sur le cadre législatif fixé par les lois de 2003 et de 2005, établissant la possibilité de négocier des «accords de méthode » par les sections syndicales d'entreprise en vue d'aménager les procédures de licenciement collectif, c'est-àdire les conditions de fonctionnement du comité d'entreprise. Les procédures classiques d'information-consultation du comité d'entreprise se trouvent ainsi intriquées dans une démarche de négociation syndicale qui, dans l'ensemble, implique des acteurs proches voire identiques lorsque les élus du comité sont syndiqués. La recherche de «bases informationnelles » au fondement de l' «approche par les capacités » est ainsi complétée par la négociation d'accords fixant plus clairement la participation des représentants du personnel au choix collectif que constitue une restructuration. Elle ne signifie pas que le choix collectif 
porte sur la désignation des salariés licenciés, mais sur la liberté,ouverte à un ensemble plus large de salariés, de choisir le départ en fonction de projets personnels dont la réalisation, sur la base de l'accord, demeure sous le contrôle des responsables syndicaux à travers leur participation aux cellules de reclassement.

\subsection{La redéfinition de l'entreprise comme expression de la « capacité politique »}

La forte inflexion que constitue la prise en compte des «projets » individuels des salariés, ouvre la voie à une pratique de négociation en rupture avec des stratégies initiales visant à bloquer les restructurations. Elle s'accompagne, dans un nombre important de cas, d'une conflictualité moins forte, mais se fonde sur une analyse des contours de l'entreprise tenue, jusque là, pour allant de soi. A cet égard, les restructurations engagées au sein de Canal Plus au début des années 2000 nous semblent exemplaires de l'émergence de ce registre nouveau que nous avions qualifié alors de « registre transactionnel » (Didry 2007).

Dans les années 1990, la négociation d'une Unité Economique et Sociale (UES) avec le syndicat majoritaire de Canal Plus, la CFDT, sous l'impulsion du PDG, a permis de clarifier le périmètre d'une entreprise aux structures capitalistiques complexes, compte tenu notamment de l'interdictionpar les autorités audiovisuelles de voir l'actionnaire majoritaire de la chaîne premium dépasser les $51 \%$ de son capital, et de la multiplication des chaînes diffusées par satellite. En 2000, le comité d'entreprise de l'UES joue un rôle décisif dans la préservation de l'entreprise au sein du groupe Vivendi issu de la Générale des Eaux, devenu, entretemps, son actionnaire majoritaire. Pour cela, il utilise son droit à formuler un avis sur le projet de fusion de Canal Plus avec Vivendi, pour obtenir la création d'un conseil de surveillance maintenant l'autonomie de gestion de Canal Plus dans le futur groupe VivendiUniversal. La négociation de conditions de départ très favorables pour les salariés concernés par la suppression d'emplois de 2001, puis celle d'un «accord de méthode » face à la procédure de licenciement collectif engagée en 2003 suggère une forme de stratégie syndicale fondée sur la préservation de l'entité Canal Plus dans le groupe Vivendi, tout en posant les bases d'un débat sur l'emploi quand les circonstances se seraient apaisées. La défense du périmètre que circonscrit l'UES a ainsi primé sur la défense directe de l'emploi, dans un contexte où dominaient le risque d'un effondrement du groupe Vivendi dans son ensemble et celui d'un démantèlement de l'UES Canal Plus.

Cette stratégie constitue une première illustration de ce registre que constitue, pour nous, l' «approche par les capacités » : elle porte non seulement sur le devenir des capacités 
des salariés touchés par les suppressions d'emplois, mais intègre également le devenir de l'entreprise elle-même, en liant préservation de la liberté individuelle des salariés et préservation du collectif de travail constitutif, à travers l'UES, de l'entité Canal Plus. Une telle stratégie se retrouve dans le cas de la chaîne de radio Europe 1, au sein du groupe Lagardère, dans le cadre de la restructuration engagée par la direction du groupe en janvier 2007 telle que l'analyse Renoux (2010). Cette restructuration vise à tirer partie des synergies possibles entre les activités du pôle média, notamment par une mutualisation plus poussée des « services supports » tels que la comptabilité, la documentation ou les services informatiques. Il conduit à engager des procédures de licenciement collectif au sein des deux entreprises du pôle, Hachette Filipacchi Associés qui intègre les activités magazine et Europe 1. Dans le cas d'Europe 1, la restructuration ébranle le «paternalisme charismatique » du PDG, au sein de l'Unité Economique et Sociale (créée en 2002 pour assurer une représentation commune aux salariés de la chaîne de radio, d'Europe 1 News et à ceux de Lagardère Active Management travaillant pour la chaîne). La procédure de licenciement débouche sur une négociation au terme de laquelle un accord fixe à 41 le nombre de suppressions d'emplois, en prévoyant également le passage sous CDI de 12 salariés sous CDD et en délimitant les activités nécessaires pour garantir l'autonomie de l'UES dans le groupe. Comme on le voit, la négociation porte ici non seulement sur l'emploi, mais aussi très clairement sur la définition de l'entreprise que constitue l'UES.

Dans le cas des réorganisations qui touchent le secteur bancaire, sous l'effet de l'informatisation et de la remise en cause des agences, et du rapprochement entre banque de détail et banque d'investissement, les initiatives des élus du personnel dans les banques françaises vont au-delà de la défense du périmètre de l'entreprise (Meixner 2011). Elles lient le souci d'éviter les «départs contraints » par le jeu sur les départs naturels et les préretraites, avec celui d'éviter les effets d'une filialisation de la gestion d'actifs en engageant la négociation d'UES regroupant les salariés dans des instances communes de représentation. Un des enjeux est de préserver la capacité d'action des comités d'entreprise menacée par la réduction du nombre de sites dans le cadre d'une politique de fermetures des agences et de centralisation des opérations de la banque de détail. Il est également de maintenir une forme de représentation cohérente avec une activité bancaire liant plus fortement la banque de détail comme lieu de vente de «produits financiers» et la banque d'investissement. Une telle stratégie permet également de faire face à la difficulté plus grande d'arriver à une mobilisation collective des salariés, pour jouer sur la menace d'une action en justice dans la revendication d'une UES. Le cas des activités bancaires suggèrent ainsi que «Les collectifs 
gagnent désormais en capacité politique ce qu'ils perdent en cohésion objective » (Segrestin 2012, p. 111). Il traduit une place croissante de la «capacité politique », comme capacité de peser sur les choix collectifs en participant à la définition des limites de la firme à partir de la cohérence économique que dessine l'apport en capacités des salariés.

Cette «capacité politique » qui s'exprime à travers la recherche des contours de l'entreprise en restructuration ne conduit pas nécessairement à une réponse immédiate, dans le cadre d'activités fortement internationalisées telles qu'on les rencontre au sein, par exemple, d'un groupe tel qu'Alstom. La dilution de la société Neyrpic de Grenoble au sein d'Alstom Hydro Power, «business unit » déployant ses activités à l'échelle mondiale en matière d'hydroélectricité (Béthoux, Brouté, Didry 2005) se traduit cependant par une interrogation sur la place de l'établissement grenoblois dans un ensemble plus large. Face à l'existence d'autres implantations du groupe, au Brésil ou en Chine, un des objectifs des élus du personnel est de dégager la spécificité de l'établissement grenoblois autour de fonctions de conception et d'expérimentation sur les turbines devant équiper les grands barrages dans le monde. Elle suscite une activité d'enquête, dans laquelle les informations produites ne reposent pas uniquement sur les documents comptables fournis par les directions, mais sur une observation de l'activité concrète des salariés, au travers des relations qu'ils tissent dans et hors de l'établissement.

Ainsi, le développement d'une activité d'observation menée par des représentants du personnel dans le cadre de 'commissions' instituées à la suite d'accords de méthode ou d'accord de GPEC est de nature à produire une connaissance fine des différentes réalités de l'entreprise, en poussant ces représentants à envisager, au-delà de leur établissement, la réalité d'un ensemble plus large de salariés (Didry et Jobert 2008, Fabre 2011). Sans pouvoir préciser davantage la portée d'une capacité politique qui se dessine autour de l'activité collective des salariés, il est possible également de suggérer une dimension propre de cette capacité à l'égard d'une forme de cogestion du capital discutée dans le cadre de débats actuels sur 1'entreprise(Benhamou 2010, Bernardins 2011, Hatchuel et Segrestin 2012). Cette capacité se dessine dans des situations de groupes financiers, où la propriété du capital tend à être assumée à un niveau plus large que celui de l'entreprise. La participation de salariés au capital du groupe ne résout pas la question de la place des salariés dans l'entreprise. Dans le cas du Monde, la position dominante de la société des rédacteurs du Monde au sein du groupe tend même à faire peser la recherche d'une rigueur financière pour faire face aux pertes, sur le personnel des autres titres du groupe. C'est paradoxalement la remise en cause de la direction du groupe par la société des rédacteurs, à l'occasion de l'entrée de nouveaux actionnaires,qui 
suscite une reconnaissance nouvelle du poids des comités d'entreprise dans le devenir des journaux contrôlés par le groupe (Dupuy 2011). En tout état de cause, la reconnaissance d'une capacité des salariés dans l'entreprise est de nature à apporter un regard nouveau sur l'entreprise en intégrant la dimension constitutive du travail à l'égard de l'entreprise comme collectivité humaine, sans confondre 'entreprise' et 'société'(au sens du droit des affaires) comme organisation juridique de la propriété du capital.

\section{Conclusion}

Si le travail apparaît comme un élément important dans l' «approche par les capacités » que Sen entend mettre au centre du débat public dans une société démocratique, cela tient au constat de la diversité des dommages que son absence, le chômage, produit sur la capacité des individus. Mais cette approche indirecte du travail, à partir de la privation d'emploi du chômeur ne doit pas occulter sa dimension collective, non seulement dans la coopération entre les salariés, mais aussi dans les modalités de «choix social » qui, pour Sen lui-même, sont attachées à l'existence de groupes humains. Si, dans un régime de liberté, les rapports de travail tendent à s'organiser autour du cadre de référence que constitue le contrat de travail, cette dimension collective revêt une forme spécifique à partir d'un engagement des individus dans le travail, en liant la liberté de choix individuel à la liberté de choix collectif qui se fait jour à travers l'existence d'institutions représentatives du personnel dans un pays tel que la France et, plus généralement, à l'échelle européenne. C'est dans ce cadre que se dessine une forme de choix collectif, autour, prioritairement, de procédures d'informationconsultation des représentants du personnel s'articulant progressivement à une activité de négociation syndicale. En mettant à l'épreuve l'existence des collectifs qui se dessinent dans le travail, les restructurations constituent la base d'une «approche par les capacités » en liant liberté de choix individuel des salariés et liberté de choix collectif, faisant apparaître une « capacité politique ».

Cette «capacité politique » dans le travail consiste en une interrogation portant simultanément sur le devenir des salariés et celui de l'entreprise, pour appréhender l'entreprise à partir de l' «apport en capacités de ses salariés ». Son identification à travers sa reconnaissance par le droit du travailet la pratique des représentants des salariés, est une étape cruciale, avant de mettre au jour les limites qu'elle rencontre et les conséquences qui peuvent en résulter, par exemple, sous la forme d'une démotivation des salariés et d'un malaise croissant au travail. Elle conduit à voir les restructurations comme des moments 
d' «épreuve » dans lesquels se joue la réalité même de l'entreprise. Face à une conception institutionnelle de l'entreprise tenant pour une évidence son incarnation par un «chef d'entreprise », elle suggère de concevoir le «pouvoir du travail » (Bélanger et Thuderoz 2010) comme un facteur constitutif de celle-ci. A cet égard, une sociologie de la «capacité politique » des travailleurs et de leurs représentants constitue le préalable d'une «politique des capacités » (Salais et Villeneuve 2004) visant à influer les choix législatifs à venir.

\section{Références bibliographiques}

Adam G. et Reynaud J-D., 1978. - Conflits du travail et changement social, Paris, Presses Universitaires de France.

Becker H.S., 2006 [1960]. - « Notes sur le concept d'engagement », Tracés n 11/1, p. $177-192$.

Bélanger J. et Thuderoz C., 2010. - «Le répertoire de l'opposition au travail ». Revue Française de Sociologie, 51-3, p. 427-460.

Benhamou S., 2010. - Améliorer la gouvernance d'entreprise et la participation des salariés, Paris, Conseil d'Analyse Stratégique, juin.

Berlin I., 1969. - Four Essays on Liberty, Oxford, Oxford University Press.

Bernardins Collège des., 2011. - L'entreprise: formes de la proprité et responsabilités sociales(documents introductifs au colloque), Collège des Bernardins 29-30 avril.

Bessy C., 2008. - «Pratiques contractuelles et individualisation de la rupture de la relation de travail »,Travail et Emploi $\mathrm{n}^{\circ} 114$, avril-juin, p. 71-86.

Béthoux E., Didry C. et Brouté R., 2006. - De l'Europe au territoire : information, consultation et mobilisations des travailleurs dans les restructurations d'Alstom, document EUROCAP.

Boltanski L., 2009. - De la critique, précis de sociologie de l'émancipation, Paris, Gallimard.

Bonvin J.-M., 2008. - « Capacités et démocratie », in De Munck J. et Zimmermann B., La liberté au prisme des capacités, Amartya Sen au-delà du libéralisme, Raisons Pratiques, Paris, Editions de l'Ecole des Hautes Etudes en Sciences Sociales, p. 237-262.

Boudon R., 1999. - Le sens des valeurs, Paris, Presses Universitaires de France.

Borzeix A. et Rot G, 2010. - Genèse d'une discipline naissance d'une revue, Sociologie du travail, Nanterre, Presses de Paris-Ouest. 
Bourdieu P., 1977. - Algérie 60, structures économiques et structures temporelles, Paris, Editions de Minuit.

Da Costa I. et Rehfeldt U., 2010, «Restructurations et comités d'entreprise européens: une dynamique de négociation transnationale», in Didry C. et Jobert A. L'entreprise en restructuration, mobilisations collectives et dynamiques institutionnelles, Rennes, Presses Universitaires de Rennes, p. 249-262.

Deakin S. et Koukiadaki A., 2012. - « Social Dialogue in the UK: Moving beyond the single channel of representation? », in De Munck J., Didry C., Ferreras I. et Jobert A., Social Dialogue and Civil Society in Europe, a New Path toward Democratic Deliberation?, Bruxelles, Peter Lang, à paraître.

De Munck J., 2008. - «Qu'est-ce qu'une capacité ?», in De Munck J. et Zimmermann B., La liberté au prisme des capacités, Amartya Sen au-delà du libéralisme, Raisons Pratiques, Paris, Editions de l'Ecole des Hautes Etudes en Sciences Sociales, p. 2150.

De Munck J. Ferreras I. et Wernerus S., 2010. - Capability for Voice et restructuration industrielle, la restructuration VW-Audi de Bruxelles en 2006-2007, document CAPRIGHT.

Desrosières A., 1993. - La politique des grands nombres, histoire de la raison statistique, Paris, La découverte.

Didry C., 1998. - «Les comités d'entreprise face aux licenciements collectifs, trois registres d'argumentation », Revue Française de Sociologie, 39/3, p. 495-534.

Didry C. et Jobert A., 2008. - Les accords de méthode en matière de restructurations : un nouveau champ du dialogue social, Rapport pour la Délégation Générale à l'Emploi et à la Formation Professionnelle.

Didry C. et Jobert A., 2010. - L'entreprise en restructuration, dynamiques institutionnelles et mobilisations collectives, Rennes, Presses Universitaires de Rennes.

Dupuy C., 2011. - «L'entreprise de presse en conflit. Libération et Le Monde en restructuration », Travail et Emploi, n 124 , p. 29-42.

Durand P., 1947. Traité de droit du travail, Paris, Dalloz.

Durkheim E., 1950. - Leçons de sociologie, Paris, Presses Universitaires de France.

Durkheim E., 1975. - «Apports de la sociologie à la psychologie et à la philosophie ». Textes, t. 1, éléments d'une théorie sociale, Paris, Editions de Minuit, p. 184188. 
Eymard-Duvernay F., 2008 - «Le marché est-il bon pour les libertés ? in De Munck J. et Zimmermann B., La liberté au prisme des capacités, Amartya Sen au-delà du libéralisme, Paris, Editions de l'Ecole des Hautes Etudes en Sciences Sociales, p. 185-214.

Fabre A., 2011. - Le contenu des accords de gestion de l'emploi, Noisy le Grand, IRES.

Hatchuel A. et Segrestin B., 2012. - Réinventer l'entreprise, la création de richesses au service du progrès collectif, Paris, Seuil.

Jeammaud A., 1989. - «Les polyvalences du contrat de travail », in Les transformations du droit du travail, études offertes à Gérard Lyon-Caen, Paris, Dalloz, p. 299-315.

Jobert A., 2008. - «La négociation collective des restructurations en France : de la consultation des représentants du personnel à la négociation des accords de méthode », Management international, 12, p. 59-66.

Koster J.-V., 2011. - «Rentrer-rester-progresser : vers une régulation européenne des parcours professionnels ? Analyse de l'accord-cadre sur les «marchés du travail inclusifs » », Communication au colloque «Emploi, compétences et relations professionnelles: quelles dynamiques de régulation aujourd'hui ? », Paris-Dauphine (24-25 janvier).

Laufer J., 2003. - «Entre égalité et inégalités : les droits des femmes dans la sphère professionnelle », L'année sociologique, vol. 53, n 1, p. 143-173.

Lefresne F. et Sauviat C., 2010. - «Restructurations au sein d'un groupe multinational : la mise à l'épreuve des relations professionnelles », in Didry C. et Jobert A. L'entreprise en restructuration, mobilisations collectives et dynamiques institutionnelles, Rennes, Presses Universitaires de Rennes, p. 221-234.

Marx K., 1993. - Le Capital, livre 1 (Traduction coordonnée par Lefebvre J.-P.), Paris, Presses Universitaires de France.

Mathieu L., 2012. - «Action collective », in A. Bévort, A. Jobert, M. Lallement et A. Mias, Dictionnaire du travail, Paris, PUF, p. 1-6.

Meixner M., 2010. - «Représentation des salariés et co-construction de l'entreprise, ne analyse comparée des dynamiques de restructuration dans le secteur bancaire (France, Allemagne, Royaume-Uni) », L'Homme et la Société, n¹75, p. 197-224.

Pharo P., 1991. - «Le droit ordinaire comme morale du commerce civil », in F. Chazel et J. Commaille (éd.), Normes juridiques et régulation sociale, Paris, Librairie générale de droit et de jurisprudence, p. 243-255. 
Renoux J.-L., 2010. - «Restructurations et régulations des emplois dans les médias du groupe Lagardère », in in Didry C. et Jobert A. L'entreprise en restructuration, mobilisations collectives et dynamiques institutionnelles, Rennes, Presses Universitaires de Rennes, p. 153-168.

Runciman W.G. et Sen A.K., 1965. «Games, Justice and the General Will », Mind, New Series, vol. 74/ 296 (Oct.), pp. 554-562.

Salais R., 2008. - «Capacités, base informationnelle et démocratie délibérative, le (contre-)exemple européen », in De Munck J. et Zimmermann B., La liberté au prisme des capacités, Amartya Sen au-delà du libéralisme, Raisons Pratiques, Paris, Editions de 1'Ecole des Hautes Etudes en Sciences Sociales, p. 297-329.

Salais R. et Villeneuve R., 2004. - Europe and the Politics of Capabilities, Cambridge, Cambridge University Press.

Segrestin D., 2012. - «Communauté de travail », in Bévort A., Jobert A., Lallement M. et Mias A., Dictionnaire du travail, Paris, Presses Universitaires de France, p. 107-113.

Sen A., 1997. - «L'inégalité, le chômage et l'Europe d'aujourd'hui », Revue internationale du Travail, vol. 136/2, p. 169-185.

Sen A., 1999. - «La possibilité du choix social», Revue de l’OFCE n70, p. 7-61 (discours de réception Prix Nobel 1998).

Sen A., 2000. - «Travail et droits », Revue internationale du travail, vol. 139/2, p. 129-139.

Sen, A., 2003a. - Un nouveau modèle économique, développement, justice, liberté. Odile Jacob. Paris.

Sen A., 2003b. - L'économie est une science morale, Paris, La Découverte.

Sen A., 2009. - «From social choice to development : the influence of Nicolas de Condorcet and Jean-Charles Borda », Revue Tiers monde, $\mathrm{n}^{\circ} 198$, avril-juin, p. 263-268.

Sen A., 2010. - L'idée de justice, Paris, Flammarion.

Sen A., 2011. - « Social Choice and the Idea of Justice », Toulouse, conférence donné au cours des journées de l'Association Charles Gide, Justice and Economics, 16 juin.

Zimmermann B., 2011. - Ce que travailler veut dire, une sociologie des capacités et des parcours professionnels, Paris, Economica, coll. Etudes sociologiques. 